\title{
Shape Evolution in Neutron-Rich Krypton Isotopes Beyond $N=60$ : First Spectroscopy of ${ }^{98,100} \mathrm{Kr}$
}

F. Flavigny, ${ }^{1,}$ P. Doornenbal, ${ }^{2}$ A. Obertelli, ${ }^{3,2}$ J.-P. Delaroche,${ }^{4}$ M. Girod,${ }^{4}$ J. Libert, ${ }^{4}$ T. R. Rodriguez, ${ }^{5}$ G. Authelet, ${ }^{3}$ H. Baba, ${ }^{2}$ D. Calvet, ${ }^{3}$ F. Château, ${ }^{3}$ S. Chen, ${ }^{2,6}$ A. Corsi, ${ }^{3}$ A. Delbart, ${ }^{3}$ J.-M. Gheller, ${ }^{3}$ A. Giganon, ${ }^{3}$ A. Gillibert, ${ }^{3}$ V. Lapoux,${ }^{3}$ T. Motobayashi, ${ }^{2}$ M. Niikura, ${ }^{7}$ N. Paul, ${ }^{2,3}$ J.-Y. Roussé, ${ }^{3}$ H. Sakurai, ${ }^{2,7}$ C. Santamaria, ${ }^{3}$ D. Steppenbeck, ${ }^{2}$ R. Taniuchi, ${ }^{2,7}$ T. Uesaka, ${ }^{2}$ T. Ando, ${ }^{2,7}$ T. Arici, ${ }^{8}$ A. Blazhev, ${ }^{9}$ F. Browne, ${ }^{10}$ A. Bruce, ${ }^{10}$ R. Carroll, ${ }^{11}$ L. X. Chung, ${ }^{12}$ M. L. Cortés,${ }^{8,13, \dagger}$ M. Dewald, ${ }^{9}$ B. Ding ${ }^{14}$ S. Franchoo, ${ }^{1}$ M. Górska ${ }^{8}$ A. Gottardo, ${ }^{1}$ A. Jungclaus, ${ }^{15}$ J. Lee, ${ }^{16}$ M. Lettmann, ${ }^{13}$ B. D. Linh,${ }^{12}$ J. Liu, ${ }^{16}$ Z. Liu, ${ }^{14}$ C. Lizarazo, ${ }^{8,13}$ S. Momiyama, ${ }^{2,7}$ K. Moschner, ${ }^{9}$ S. Nagamine, ${ }^{7}$ N. Nakatsuka, ${ }^{17}$ C. Nita, ${ }^{18}$ C. R. Nobs,${ }^{10}$ L. Olivier, ${ }^{1}$ R. Orlandi, ${ }^{19}$ Z. Patel, ${ }^{11}$ Zs. Podolyák, ${ }^{11}$ M. Rudigier, ${ }^{11}$ T. Saito, ${ }^{7}$ C. Shand, ${ }^{11}$ P. A. Söderström, ${ }^{2}$

I. Stefan, ${ }^{1}$ V. Vaquero, ${ }^{15}$ V. Werner, ${ }^{13} \mathrm{~K}$. Wimmer, ${ }^{7}$ and $\mathrm{Z}$. $\mathrm{Xu}^{16}$

${ }^{1}$ Institut de Physique Nucléaire, CNRS-IN2P3, Université Paris-Sud, Université Paris-Saclay, 91406 Orsay Cedex, France

${ }^{2}$ RIKEN Nishina Center, 2-1 Hirosawa, Wako, Saitama 351-0198, Japan

${ }^{3} I R F U, C E A$, Université Paris-Saclay, F-91191 Gif-sur-Yvette, France

${ }^{4} C E A, D A M, D I F$, F-91297 Arpajon, France

${ }^{5}$ Departamento de Física Teórica, Universidad Autónoma de Madrid, E-28049 Madrid, Spain

${ }^{6}$ School of Physics and State Key Laboratory of Nuclear Physics and Technology,

Peking University, Beijing 100871, People's Republic of China

${ }^{7}$ Department of Physics, University of Tokyo, 7-3-1 Hongo, Bunkyo, Tokyo 113-0033, Japan

${ }^{8}$ GSI Helmholtzzentrum für Schwerionenforschung GmbH, 64291 Darmstadt, Germany

${ }^{9}$ Institut für Kernphysik, Universität zu Köln, 50937 Köln, Germany

${ }^{10}$ School of Computing Engineering and Mathematics, University of Brighton, Brighton BN2 4GJ, United Kingdom

${ }^{11}$ Department of Physics, University of Surrey, Guildford GU2 7XH, United Kingdom

${ }^{12}$ Institute for Nuclear Science and Technique, VINATOM, 179 Hoang Quoc Viet, Cau Giay, Hanoi, Vietnam

${ }^{13}$ Institut für Kernphysik, Technische Universität Darmstadt, 64289 Darmstadt, Germany

${ }^{14}$ Institute of Modern Physics, Chinese Academy of Sciences, Lanzhou 730000, People's Republic of China

${ }^{15}$ Instituto de Estructura de la Materia, CSIC, 28006 Madrid, Spain

${ }^{16}$ Department of Physics, University of Hong Kong, Pokfulam, Hong Kong

${ }^{17}$ Department of Physics, Faculty of Science, Kyoto University, Kyoto 606-8502, Japan

${ }^{18}$ Horia Hulubei National Institute of Physics and Nuclear Engineering (IFIN-HH), RO-077125 Bucharest, Romania

${ }^{19}$ Advanced Science Research Center, Japan Atomic Energy Agency, Tokai, Ibaraki, 319-1195, Japan

(Received 22 February 2017; published 14 June 2017)

We report on the first $\gamma$-ray spectroscopy of low-lying states in neutron-rich ${ }^{98,100} \mathrm{Kr}$ isotopes obtained from ${ }^{99,101} \mathrm{Rb}(p, 2 p)$ reactions at $\sim 220 \mathrm{MeV} /$ nucleon. A reduction of the $2_{1}^{+}$state energies beyond $N=60$ demonstrates a significant increase of deformation, shifted in neutron number compared to the sharper transition observed in strontium and zirconium isotopes. State-of-the-art beyond-mean-field calculations using the Gogny D1S interaction predict level energies in good agreement with experimental results. The identification of a low-lying $\left(0_{2}^{+}, 2_{2}^{+}\right)$state in ${ }^{98} \mathrm{Kr}$ provides the first experimental evidence of a competing configuration at low energy in neutron-rich krypton isotopes consistent with the oblate-prolate shape coexistence picture suggested by theory.

DOI: 10.1103/PhysRevLett.118.242501

Sudden variations of nuclear properties as a function of nucleon number lie at the heart of our understanding of atomic nuclei. The nuclear-shell model was itself motivated by the discontinuities observed in two-nucleon separation energies and radii at specific (magic) nucleon numbers $[1,2]$. Similarly, the presence of a rapid onset of deformation and change of equilibrium shape when adding only one or a few nucleons drives the modeling of collectivity as it highlights the interplay between macroscopic properties and microscopic degrees of freedom. Related to the competition between the sphericity-favoring pairing interaction and the deformation-driving neutron-proton interaction [3], such a rapid growth of collectivity can be described in different frameworks. In the context of shape coexistence [4], an abrupt change of ground-state properties can be interpreted as stemming from the crossing of two distinct quantum configurations of nucleons coexisting at low excitation energy. An intruder configuration, such as a multiparticle-multihole excitation above a closed shell or subshell, can, for example, profit from residual interactions with other nucleons to become energetically favorable. Another interpretation consists of describing this 
drastic shape change as a quantum phase transition [5], and transitional nuclei as critical points described using dynamical symmetries [6,7]. The differences between these two mechanisms of shape change are subtle [8], and debates persist on the modeling of transitional regions $[9,10]$.

Across the nuclear chart, one of the most drastic and rapid shape transitions appears in the $A \simeq 100$ region at neutron number $N=60$ for neutron-rich zirconium and strontium isotopes $[4,11]$. The main experimental signatures for this shape change come from ground-state properties, namely, a discontinuity in binding energies [12] and an increase in charge radii [13], but also from the spectroscopy of low-lying $2_{1}^{+}$states showing a sudden drop in energy [14]. In addition, the shape change is accompanied by the gradual lowering of an excited $0^{+}$state $[15,16]$, interpreted as the bandhead of a deformed configuration finally crossing with the spherical ground-state band at $N=60$. As opposed to other regions of shape coexistence, all the detailed studies of transition amplitudes between low-lying states in ${ }^{98} \mathrm{Sr}$ [17-19] and ${ }^{100} \mathrm{Zr}$ [20-22] point to a weak mixing $(\leq 20 \%)$ between the two unperturbed configurations in the $0^{+}$states, compatible with the picture of a sudden structural change. Recently, shape coexistence was also directly established away from the $N=60$ transition in ${ }^{94,96} \mathrm{Zr}[23,24]$, pointing to a more widespread phenomenon in the region and to the importance of the $Z=40$ subshell closure in this respect. More specifically, largescale Monte Carlo shell-model predictions [25] suggested that the deformed $0^{+}$states in ${ }^{98,100} \mathrm{Zr}$ isotopes arise from a sizable excitation of protons to the $0 g_{9 / 2}$ orbital across the $Z=40$ subshell closure together with a close grouping of neutron effective single-particle energies, both mediated by the tensor and central forces. Interestingly, the sudden onset of deformation at $N=60$ in the zirconium and strontium chains was not observed for ${ }^{96} \mathrm{Kr}$ [26]. Instead, a smooth reduction of $E\left(2_{1}^{+}\right)$energy and the rise of $B\left(E 2,0_{1}^{+} \rightarrow 2_{1}^{+}\right)$ excitation strength suggest a gradual development of collectivity. Moreover, no low-lying intruder states have been observed yet around $N=60$ for krypton isotopes; i.e., no clear evidence of shape coexistence exists. Mass measurements of ${ }^{96,97} \mathrm{Kr}$ [27], and ${ }^{98,100} \mathrm{Rb}$ [28] isotopes together with charge radii studies [29,30] also demonstrated that this abrupt $N=60$ shape transition extends down to $Z=37$ and not to $Z=36$ in ${ }^{96} \mathrm{Kr}$. However, in the unexplored $N>60$ region for krypton isotopes, one cannot exclude that a few additional neutrons could trigger a shape transition between prolate and oblate deformed configurations, predicted to compete at low excitation energies [28,31-34]. In this Letter, we present the spectroscopy of very neutron-rich ${ }^{98,100} \mathrm{Kr}$ nuclei and characterize for the first time the evolution of collectivity beyond $N=60$ in krypton isotopes.

The experiment was performed at the Radioactive Isotope Beam Factory at the RIKEN Nishina Center.
The in-flight fission of a ${ }^{238} \mathrm{U}$ beam with a mean intensity of $27 \mathrm{pnA}$ accelerated to $345 \mathrm{MeV} /$ nucleon was induced by the collision with a 3 -mm-thick ${ }^{9} \mathrm{Be}$ primary target at the object point of the BigRIPS separator [35]. Purification of the secondary beam was performed using $\mathrm{Al}$ degraders at the $F 1$ and $F 5$ dispersive planes (5- and 2-mm thick). The secondary cocktail beam containing $6.4 \%$ of ${ }^{99} \mathrm{Rb}$ and $0.4 \%$ of ${ }^{101} \mathrm{Rb}$ at respective averaged rates of 220 and $16 \mathrm{~s}^{-1}$ impinged for 29 hours on a 99(1)-mm-thick liquid hydrogen target of $73.22(8) \mathrm{kg} / \mathrm{m}^{3}$ density cooled to $20 \mathrm{~K}$. Event-by-event identification of projectiles and reaction residues in atomic number $(Z)$ and mass-over-charge $(A / Q)$ ratio was achieved using the TOF- $B \rho-\Delta E$ method [35] in both BigRIPS and ZeroDegree spectrometers. Radioactive isotopes of ${ }^{98,100} \mathrm{Kr}$ were produced via the $(p, 2 p)$ direct reactions from ${ }^{99,101} \mathrm{Rb}$ at energies ranging from $\sim 260$ to $\sim 170 \mathrm{MeV} /$ nucleon, respectively, at the entrance and exit of the target.

The MINOS time-projection chamber [36] surrounding the target cell was used to reconstruct the reaction vertex position using the measured tracks of the outgoing protons. The reconstructed vertex was defined as the middle of the segment of closest approach between two trajectories, either from the two outgoing protons or from one proton and the beam particle tracked through two upstream position-sensitive parallel-plate avalanche counters [37]. The detection efficiency of at least one proton was simulated at $95 \%$ with a vertex position resolution of $5 \mathrm{~mm}$ (FWHM) along the beam axis [36]. The DALI2 high-efficiency gamma spectrometer [38] surrounded the MINOS device to detect in-flight deexcitation $\gamma$ rays of ${ }^{98,100} \mathrm{Kr}$. It was composed of $186 \mathrm{NaI}(\mathrm{Tl})$ crystals calibrated using ${ }^{88} \mathrm{Y},{ }^{60} \mathrm{Co},{ }^{133} \mathrm{Ba}$, and ${ }^{137} \mathrm{Cs}$ sources down to $356 \mathrm{keV}$. Add-back was applied when the centers of hit detectors were less than $15 \mathrm{~cm}$ apart. A full simulation of the array was performed with the GEANT4 toolkit [39] to extract response functions. The simulations included (i) individual crystal thresholds set at $100 \mathrm{keV}$ on average, (ii) individual energy resolutions obtained using the calibration sources, (iii) experimental velocity distributions of projectiles from BigRIPS, and (iv) the lifetime of the decaying state. This simulation resulted in a full-energy peak detection efficiency of $41 \%$ for $500-\mathrm{keV} \gamma$ rays emitted in flight along the target at relative velocity $\beta=v / c$ ranging from 0.62 to 0.54 . Other beam-energy losses through materials along the line were taken into account for the beam velocity determination and benchmarked using an empty target measurement (relative agreement of $0.2 \%$ ). Moreover, the full analysis procedure including Doppler correction and lifetime effects in the simulated response functions was validated on ${ }^{94} \mathrm{Kr}$ for which the $2_{1}^{+} \rightarrow 0_{g s}^{+}$transition energy was extracted with a $2-\mathrm{keV}$ deviation from published values $[26,40]$, well within experimental uncertainties.

Doppler-corrected $\gamma$-ray spectra of ${ }^{98,100} \mathrm{Kr}$ are presented in Fig. 1 together with least-squares fits including simulated 


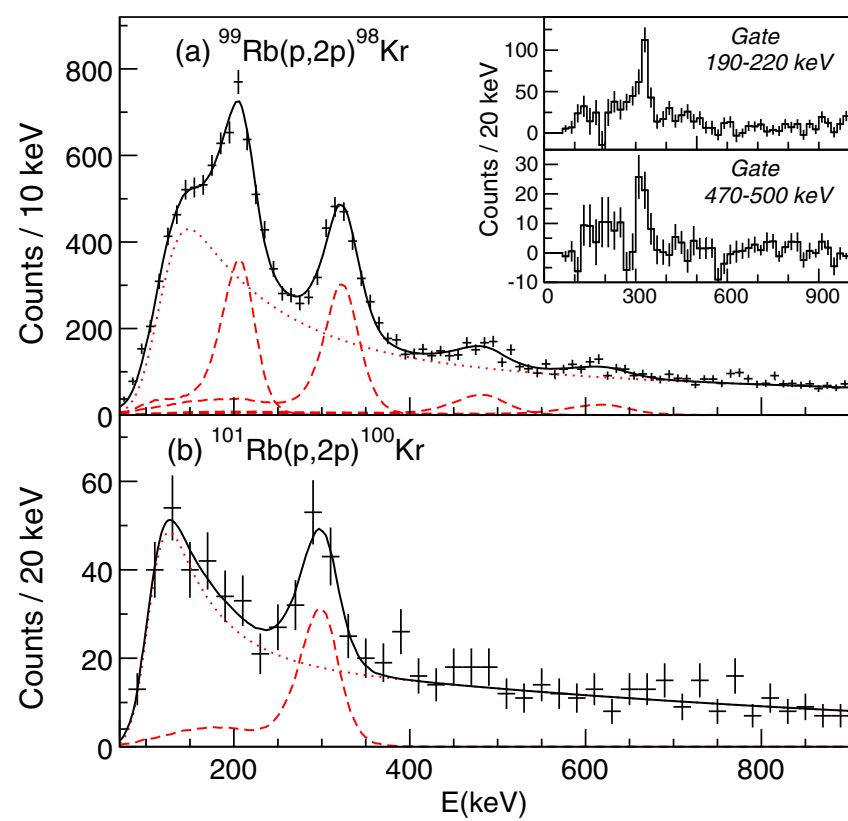

FIG. 1. Doppler-corrected $\gamma$ spectrum of ${ }^{98} \mathrm{Kr}$ (top) and ${ }^{100} \mathrm{Kr}$ (bottom) produced from ${ }^{99,101} \mathrm{Rb}(p, 2 p)$ reactions. For each spectrum, the global fit (solid black line) includes individual simulated responses (red dashed lines) and a two-component exponential background (red dotted line). Add-back was applied and $\gamma$ multiplicities below 6 were considered. The inset of the top panel shows background-subtracted $\gamma-\gamma$ coincidence spectra for ${ }^{98} \mathrm{Kr}$.

response functions of identified transitions and a two-component exponential background. This background was folded with a step function to account for the lowenergy cutoff. Starting with ${ }^{98} \mathrm{Kr}$ in Fig. 1(a), four peaks are visible on the energy spectrum and each one was fitted using a set of response functions assuming different energies and lifetimes. This procedure led to transition energies of 216(10), 329(7), 498(13), and 638(25) keV. Uncertainties are dominated by systematic errors from lifetime effects estimated using upper limits from $\chi^{2}$ profiles but also include a statistical contribution from the fit and a calibration error $(4 \mathrm{keV})$ in the considered energy range. With respect to the $329-\mathrm{keV}$ transition, the intensity of the $216-\mathrm{keV} \gamma$ ray extracted from the peak shape is significant but uncertain (between 50\% and 120\%) due to the ambiguity induced by the unknown shape of the low-energy background. Background-subtracted $\gamma-\gamma$ coincidence spectra gated on the peaks corresponding to the 216- and 498-keV transitions are shown in the inset of Fig. 1(a). These two transitions are in coincidence with the $329-\mathrm{keV}$ transition but not between themselves. Based on these coincidences and in line with $\gamma$-ray intensities, the $329-\mathrm{keV}$ transition was assigned to the $2_{1}^{+} \rightarrow 0_{1}^{+}$deexcitation with its two coincident $\gamma$ rays on top as shown in the proposed level scheme in Fig. 2(b). The weak $638-\mathrm{keV}$ transition, enhanced in the ${ }^{100} \mathrm{Rb}(p, 2 p n){ }^{98} \mathrm{Kr}$ channel, was not seen in coincidence with any of the other $\gamma$ rays.

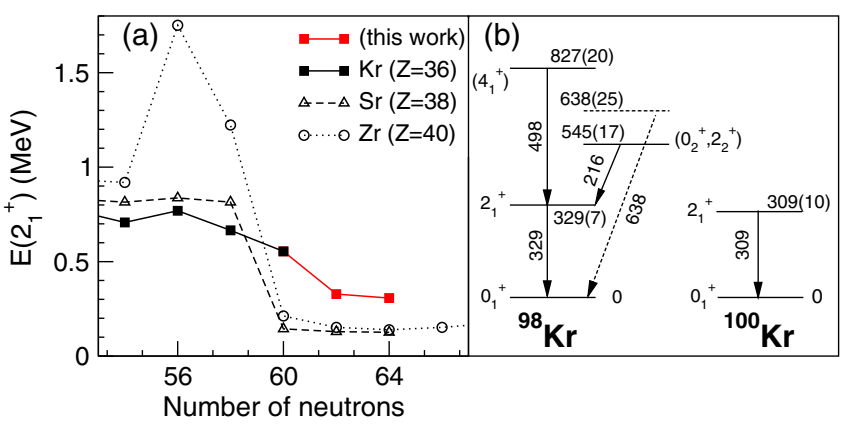

FIG. 2. (a) Systematics of experimental data for $E\left(2_{1}^{+}\right)$. Data points from this Letter are highlighted in red. Error bars are smaller than the marker size. (b) Experimental level schemes for ${ }^{98,100} \mathrm{Kr}$.

Therefore, it may be a transition to the ground state as indicated by the dashed line in Fig. 2(b). For the $545-\mathrm{keV}$ state, two spin-parity hypotheses are compatible with the nonobservation of a transition to the ground state and with a $216-\mathrm{keV}$ transition short-lived enough to be measured in this experiment: (i) either a $0_{2}^{+}$state with a large $B\left(E 2,0_{2}^{+} \rightarrow 2_{1}^{+}\right)$or (ii) a $2_{2}^{+}$state decaying via a M1-dominated transition to the $2_{1}^{+}$state. In addition, a $4_{1}^{+}$spin-parity has been discarded for this state because it would lead to a $E\left(4_{1}^{+}\right) / E\left(2_{1}^{+}\right)$ratio of $\sim 1.6$ typical of rigid spherical nuclei, very unlikely in such a midshell region. For the $827-\mathrm{keV}$ state, a $4_{1}^{+}$tentative assignment is favored by this experiment based on the nonobservation of a decay to the ground state and on relative intensities of the $4_{1}^{+} \rightarrow 2_{1}^{+}$and $2_{1}^{+} \rightarrow 0_{1}^{+}$transitions populated by $(p, 2 p)$ reactions to other nuclei studied in the same conditions [41-43]. Following the same procedure for ${ }^{100} \mathrm{Kr}$ [Fig. 1(b)], only one transition at 309(10) $\mathrm{keV}$ was observed and assigned to the $2_{1}^{+} \rightarrow 0_{1}^{+}$transition.

Our results extend the $E\left(2_{1}^{+}\right)$trend shown in Fig. 2(a) up to $N=64$ for the krypton isotopes. While a rather flat behavior persisted up to $N=60$ with previously measured values, our work indicates a significant drop in energy of $40 \%$ at $N=62$ and a stabilization afterwards. Compared to $\mathrm{Sr}$ and $\mathrm{Zr}$, the overall trend behaves more smoothly and a reduced but marked decrease in energy is shifted to $N=62$. In addition, the identification of a $\left(0_{2}^{+}, 2_{2}^{+}\right)$state at $545(17) \mathrm{keV}$ in ${ }^{98} \mathrm{Kr}$ close to the $2_{1}^{+}$state is the first experimental evidence for a coexisting band intruding at low energy in neutron-rich krypton isotopes. These observations point to structural changes around $N=62$. Are they manifestations of an underlying shape transition somehow analogous to the ones observed in strontium and zirconium isotopes?

To gain insight into this subtle evolution, we used the five-dimensional collective Hamiltonian (5DCH) [33] beyond-mean-field model with the Gogny D1S effective interaction $[44,45]$ to calculate energy levels of neutronrich krypton isotopes and to investigate the weight of 


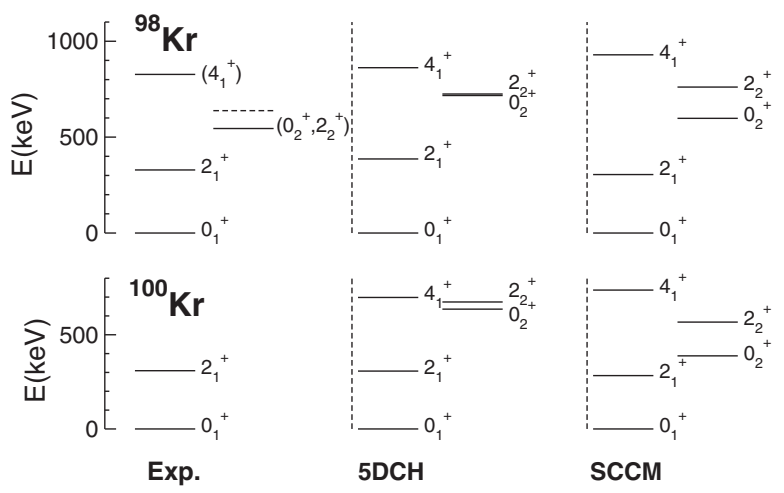

FIG. 3. Comparison between experimental and theoretical lowlying states in ${ }^{98,100} \mathrm{Kr}$ isotopes from this work.

different deformations in the composition of their collective wave functions. Such features may be anticipated, based on topology displayed by the potential energy surfaces which show deformed oblate and prolate minima separated by triaxial barriers $\sim 2.1$ and $\sim 1.6 \mathrm{MeV}$ high for ${ }^{98} \mathrm{Kr}$ and ${ }^{100} \mathrm{Kr}$, respectively. Note that the 5DCH model successfully described the shape transition from an oblate ground state in ${ }^{72} \mathrm{Kr}$ to prolate in ${ }^{76} \mathrm{Kr}$ and revealed the key role played by the triaxial degree of freedom [46].

In Fig. 3, the calculated levels for ${ }^{98,100} \mathrm{Kr}$ are compared to experiment. For both nuclei, the predicted ground-state band levels are in very good agreement with experiment (differences within $60 \mathrm{keV}$ ). More importantly, two excited levels $0_{2}^{+}$and $2_{2}^{+}$are predicted to lie between the $2_{1}^{+}$and $4_{1}^{+}$ states. Our observation of a $\left(0_{2}^{+}, 2_{2}^{+}\right)$state in ${ }^{98} \mathrm{Kr}$ at 545(17) $\mathrm{keV}$ matches well with this prediction. 5DCH probability densities in the $\beta$ and $\gamma$ quadrupole deformation coordinate space are plotted in Fig. 4 for the $0_{1}^{+}, 2_{1}^{+}, 4_{1}^{+}, 0_{2}^{+}$, and $2_{2}^{+}$states. As shown in this figure for ${ }^{98} \mathrm{Kr}$, the yrast band is predicted to start from triaxial-oblate-dominant $0_{1}^{+}$ and $2_{1}^{+}$collective wave functions, evolving toward a stabilized prolate profile in the $4_{1}^{+}$and higher-spin states (not shown). For ${ }^{100} \mathrm{Kr}$, the same transition is already foreseen from the $2_{1}^{+}$state. Such an oblate-to-prolate shape evolution inside a band structure closely resembles those studied on the proton-rich side in ${ }^{72} \mathrm{Kr}$ [47] and ${ }^{70,72} \mathrm{Se}$ [48]. Furthermore, the $0_{2}^{+}$and $2_{2}^{+}$excited states intruding below the $4_{1}^{+}$state in ${ }^{98} \mathrm{Kr}$ are predicted to originate from a prolate shape competing at low energy with that for the oblate ground state as illustrated in top panel of Fig. 4. The shapecoexistent $0_{2}^{+}$state persists at $N=64$ in ${ }^{100} \mathrm{Kr}$ but the $2_{2}^{+}$ state becomes even more triaxially spread with a collective wave function dominated by a $K=2$ projected angularmomentum component. More quantitatively, our calculations point to a rather smooth shape evolution starting in ${ }^{96} \mathrm{Kr}$ and going to ${ }^{100} \mathrm{Kr}$, reflected by (i) the progressive sign inversion of spectroscopic electric-quadrupole moments $\left(Q_{s p}\right)$ from +20 to $-62 e \mathrm{fm}^{2}$ for the $2_{1}^{+}$state and from -49 to $+27 e \mathrm{fm}^{2}$ for the $2_{2}^{+}$state, (ii) the large

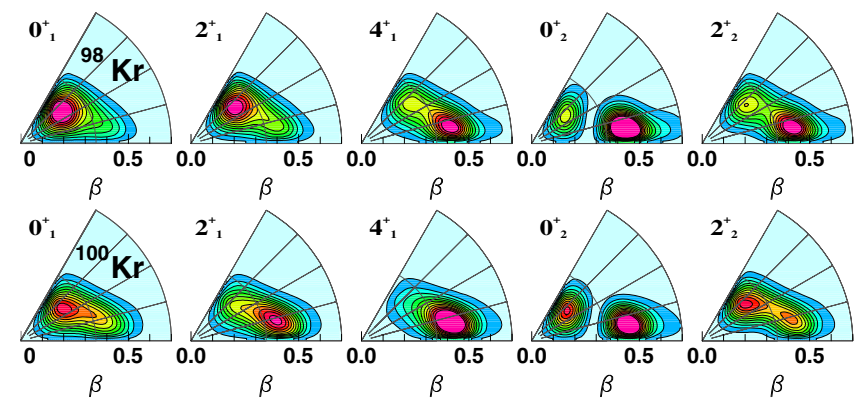

FIG. 4. Probability densities in the $\beta-\gamma$ deformation space for low-lying $0_{1}^{+}, 2_{1}^{+}, 4_{1}^{+}, 0_{2}^{+}$, and $2_{2}^{+}$collective states in ${ }^{98,100} \mathrm{Kr}$ from $5 \mathrm{DCH}$ calculations (see text for details).

interband $B\left(E 2,0_{2}^{+} \rightarrow 2_{1}^{+}\right)$values reaching 1653 and $2637 e^{2} \mathrm{fm}^{4}$ in ${ }^{98} \mathrm{Kr}$ and ${ }^{100} \mathrm{Kr}$, respectively, and (iii) a significant mixing of angular-momentum projected components $(K=0$ and $K=2)$ in the $2^{+}$states collective wave functions. Going back to the krypton isotopic chain from $N=56$ to 64 shown in Fig. 5, the calculated $E\left(2_{1}^{+}\right)$energy trend shows a smooth and moderate decrease, in overall good agreement with experimental data. The marked energy decrease observed at $N=62$ in the experiment is somewhat smoothed in the calculations as also noticed for $\mathrm{Sr}$ and $\mathrm{Zr}$ isotopes [33], a feature attributed to the lack of projection on good particle number in the $5 \mathrm{DCH}$ theory. Concerning nonyrast states, the $0_{2}^{+}$state is predicted to dive in energy continuously from $N=54$ to 60 , faster than the $2_{2}^{+}$state, so that both states lie significantly below the $4_{1}^{+}$ state at $N=62$.

To further investigate the robustness of our theoretical interpretation, we performed another beyond-mean-field calculation also using the Gogny D1S interaction but based on a symmetry-conserving configuration-mixing method (SCCM) as described in [34]. This approach relies upon the generator coordinate method in $(\beta, \gamma)$ variables within a space spanned by projected Hartree-Fock-Bogoliubov states at good angular momentum and particle number.

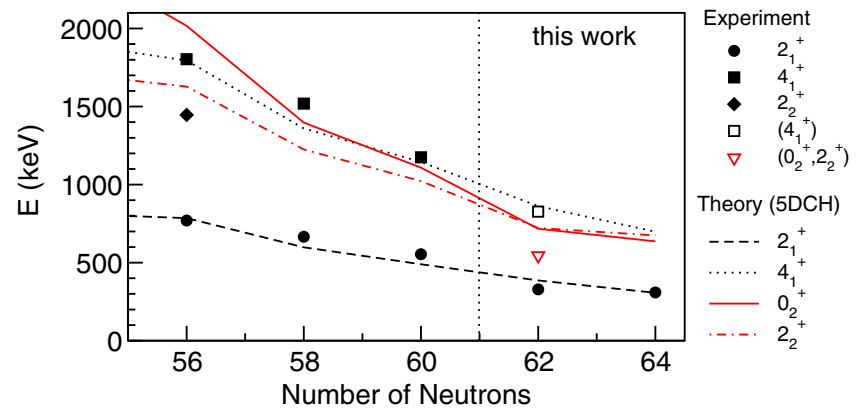

FIG. 5. Comparison between experimental and 5DCH+D1S theoretical energies of the low-lying $2_{1}^{+}, 4_{1}^{+}, 0_{2}^{+}$, and $2_{2}^{+}$states in krypton isotopes. Open symbols are for states seen experimentally for which spin-parity assignment is uncertain. Up to $N=60$, energies are taken from $[26,40,49]$. 
The level schemes obtained are also displayed in Fig. 3 for ${ }^{98,100} \mathrm{Kr}$. Agreement with experimental level energies similar to that achieved in the 5DCH approach is reached and a clear oblate-prolate shape-coexistence situation is also predicted, with a $0_{2}^{+}$state calculated at $598 \mathrm{keV}$ in ${ }^{98} \mathrm{Kr}$ close to the 545(17)-keV experimental value. Both calculations and experimental data indicate that shape coexistence might drive the evolution of low-lying states in neutron-rich krypton isotopes. Still, differences of configuration mixing exists between the two models considered and a dedicated benchmark study in the spirit of those performed for neutron-deficient krypton isotopes [50,51] would be relevant. For example, the SCCM calculations predict coexisting bands in ${ }^{98} \mathrm{Kr}$ with very little mixing quantified by an interband $B\left(E 2,0_{2}^{+} \rightarrow 2_{1}^{+}\right)$value of only $19 e^{2} \mathrm{fm}^{4}$. It also predicts larger axial deformation in general and a sharp shape transition between $N=62$ and $N=64$, with a crossing in energy of the two coexisting configurations characterized by $Q_{s p}\left(2_{1}^{+}\right)$going from +72 to $-83 e \mathrm{fm}^{2}$ for ${ }^{98} \mathrm{Kr}$ and ${ }^{100} \mathrm{Kr}$, respectively.

Finally, relative $\gamma$-ray intensities with respect to the $2_{1}^{+} \rightarrow 0_{1}^{+}$transition indicate that the ${ }^{99} \mathrm{Rb}(p, 2 p)$ reaction significantly populates the $\left(0_{2}^{+}, 2_{2}^{+}\right)$state in ${ }^{98} \mathrm{Kr}$ $[I(216)>50 \%]$. Based on recent studies of odd-even rubidium isotopes [52,53] for $N \geq 60$, the ground states of ${ }^{97,99,101} \mathrm{Rb}$ are understood as coming from a $\pi g_{9 / 2}[431] 3 / 2^{+}$Nilsson-model configuration with a large prolate deformation. The selectivity of the $(p, 2 p)$ reaction thus indicates a significant overlap between the $\left(0_{2}^{+}, 2_{2}^{+}\right)$ state in ${ }^{98} \mathrm{Kr}$ and the prolate-deformed ground state of ${ }^{99} \mathrm{Rb}$, consistent with calculations. The nonobservation of such a $\left(0_{2}^{+}, 2_{2}^{+}\right)$state in the same energy range in ${ }^{100} \mathrm{Kr}$ [ $<40 \%$ of $I\left(2_{1}^{+} \rightarrow 0_{1}^{+}\right)$at a $95 \%$ confidence level] may indicate a change in the structure of low-lying nonyrast states with respect to ${ }^{98} \mathrm{Kr}$, requiring further experimental investigations.

To conclude, we performed the first spectroscopy of krypton isotopes beyond $N=60$ and showed a significant drop of $E\left(2_{1}^{+}\right)$for ${ }^{98} \mathrm{Kr}$ further stabilized for ${ }^{100} \mathrm{Kr}$. In ${ }^{98} \mathrm{Kr}$, the additional identification of a $\left(0_{2}^{+}, 2_{2}^{+}\right)$state only $216(10) \mathrm{keV}$ above the $2_{1}^{+}$state provides the first experimental evidence of the lowering of an excited band coexisting with the ground-state one. Beyond-mean-field calculations reproduce rather well the extracted energy levels and link them to the coexistence of oblate and prolate configurations competing at low energy, which might cross around ${ }^{98,100} \mathrm{Kr}$. These observations moderate the previously established picture of neutron-rich krypton isotopes as a sharp low- $Z$ limit for the shape transition region at $N \simeq 60$ close to $A=100$, and highlight that the richness of collective behaviors observed for neutron-rich zirconium and strontium isotopes extends to krypton. This new step towards a delineation of the transitional region calls for the search of excited bands in ${ }^{94,96} \mathrm{Kr}$ to further benchmark shape evolution scenarios, since extracting intra- and interband transition probabilities via Coulomb excitation of ${ }^{98,100} \mathrm{Kr}$ represents a longer-term goal presumably requiring next-generation facilities.

We express our gratitude to the RIKEN Nishina Center accelerator staff for providing the stable and high-intensity uranium beam and to the BigRIPS team for the smooth operation of the secondary beams. The development of MINOS has been supported by the European Research Council through ERC Grant No. MINOS-258567. T. R. Rodriguez acknowledges support from GSI-Darmstadt computing facilities and the financial support of Ministerio de Economa y Competitividad under Contract No. FIS-2014-53434 and Programa Ramon y Cajal 2012 No. 11420. S. Chen acknowledges support of the IPA program at RIKEN Nishina Center. K. Moschner acknowledges support from the German BMBF Grant No. 05P15PKFNA. M. L. Cortés, M. Lettman, C. Lizarazo, and V. Werner acknowledge support from the German BMBF Grant No. 05P12RDFN8. L. X. Chung and B.D. Linh acknowledge support from the Vietnam Ministry of Science and Technology, and the Radioactive Isotope Physics Laboratory of the RIKEN Nishina Center for supporting their stay during the experiment. A. Jungclaus and V. Vaquero acknowledge support from the Spanish Ministerio de Economia y Competitividad under Contract No. FPA2014-57196-C54-P. U.K. participants acknowledge support from the Science and Technology Facilities Council (STFC).

Note added.-Recently, the energy of the $4_{1}^{+}$state in ${ }^{96} \mathrm{Kr}$ was measured in [49]. The corresponding data point has been added to Fig. 5 in this Letter after the review process. Note that for ${ }^{98} \mathrm{Kr}$, our tentative $\left(4_{1}^{+}\right)$state at $827(20) \mathrm{keV}$ leads to a $R_{42}=E\left(4_{1}^{+}\right) / E\left(2_{1}^{+}\right)$of $2.51(8)$, significantly higher than the value of 2.12(1) obtained for ${ }^{96} \mathrm{Kr}$ [49].

*flavigny@ipno.in2p3.fr

'RIKEN Nishina Center, 2-1 Hirosawa, Wako, Saitama 3510198, Japan.

[1] M. G. Mayer, Phys. Rev. 75, 1969 (1949).

[2] O. Haxel, J. H. D. Jensen, and H. E. Suess, Phys. Rev. 75, 1766 (1949).

[3] R. B. Cakirli and R. F. Casten, Phys. Rev. Lett. 96, 132501 (2006).

[4] K. Heyde and J. L. Wood, Rev. Mod. Phys. 83, 1467 (2011).

[5] P. Cejnar, J. Jolie, and R. F. Casten, Rev. Mod. Phys. 82, 2155 (2010).

[6] F. Iachello, Phys. Rev. Lett. 85, 3580 (2000).

[7] F. Iachello, Phys. Rev. Lett. 87, 052502 (2001).

[8] K. Heyde, J. Jolie, R. Fossion, S. De Baerdemacker, and V. Hellemans, Phys. Rev. C 69, 054304 (2004).

[9] R. F. Casten and N. V. Zamfir, Phys. Rev. Lett. 87, 052503 (2001).

[10] P. E. Garrett et al., Phys. Rev. Lett. 103, 062501 (2009). 
[11] S. A. E. Johansson, Nucl. Phys. 64, 147 (1965).

[12] M. Wang, G. Audi, A. H. Wapstra, F. G. Kondev, M. MacCormick, X. Xu, and B. Pfeiffer, Chin. Phys. C 36, 1603 (2012).

[13] I. Angeli and K. P. Marinova, At. Data Nucl. Data Tables 99, 69 (2013).

[14] H. Wollnik, F. K. Wohn, K. D. Wünsch, and G. Jung, Nucl. Phys. A291, 355 (1977).

[15] T. A. Khan, W. D. Lauppe, K. Sistemich, H. Lawin, G. Sadler, and H. A. Selic, Z. Phys. A 283, 105 (1977).

[16] F. Schussler, J. A. Pinston, E. Monnand, A. Moussa, G. Jung, E. Koglin, B. Pfeiffer, R. V. F. Janssens, and J. van Klinken, Nucl. Phys. A339, 415 (1980).

[17] C. Y. Wu, H. Hua, and D. Cline, Phys. Rev. C 68, 034322 (2003).

[18] J. Park et al., Phys. Rev. C 93, 014315 (2016).

[19] E. Clément et al., Phys. Rev. Lett. 116, 022701 (2016).

[20] F. K. Wohn, J. C. Hill, C. B. Howard, K. Sistemich, R. F. Petry, R. L. Gill, H. Mach, and A. Piotrowski, Phys. Rev. C 33, 677 (1986).

[21] H. Mach, M. Moszynński, R. L. Gill, F. K. Wohn, J. A. Winger, J. C. Hill, G. Molnár, and K. Sistemich, Phys. Lett. B 230, 21 (1989).

[22] C. Y. Wu, H. Hua, and D. Cline, Phys. Lett. B 541, 59 (2002).

[23] A. Chakraborty et al., Phys. Rev. Lett. 110, 022504 (2013).

[24] C. Kremer et al., Phys. Rev. Lett. 117, 172503 (2016).

[25] T. Togashi, Y. Tsunoda, T. Otsuka, and N. Shimizu, Phys. Rev. Lett. 117, 172502 (2016).

[26] M. Albers et al., Phys. Rev. Lett. 108, 062701 (2012).

[27] S. Naimi et al., Phys. Rev. Lett. 105, 032502 (2010).

[28] V. Manea et al., Phys. Rev. C 88, 054322 (2013).

[29] C. Thibault et al., Phys. Rev. C 23, 2720 (1981).

[30] M. Keim, E. Arnold, W. Borchers, U. Georg, A. Klein, R. Neugart, L. Vermeeren, R. E. Silverans, and P. Lievens, Nucl. Phys. A586, 219 (1995).

[31] P. Sarriguren, Phys. Rev. C 91, 044304 (2015).
[32] P. Möller, A. J. Sierk, R. Bengtsson, H. Sagawa, and T. Ichikawa, Phys. Rev. Lett. 103, 212501 (2009).

[33] J.-P. Delaroche, M. Girod, J. Libert, H. Goutte, S. Hilaire, S. Péru, N. Pillet, and G. F. Bertsch, Phys. Rev. C 81, 014303 (2010).

[34] T. R. Rodriguez, Phys. Rev. C 90, 034306 (2014).

[35] N. Fukuda, T. Kubo, T. Ohnishi, N. Inabe, H. Takeda, D. Kameda, and H. Suzuki, Nucl. Instrum. Methods Phys. Res., Sect. B 317, 323 (2013).

[36] A. Obertelli et al., Eur. Phys. J. A 50, 8 (2014).

[37] H. Kumagai, T. Ohnishi, N. Fukuda, H. Takeda, D. Kameda, N. Inabe, K. Yoshida, and T. Kubo, Nucl. Instrum. Methods Phys. Res., Sect. B 317, 717 (2013).

[38] S. Takeuchi, T. Motobayashi, Y. Togano, M. Matsushita, N. Aoi, K. Demichi, H. Hasegawa, and H. Murakami, Nucl. Instrum. Methods Phys. Res., Sect. A 763, 596 (2014).

[39] S. Agostinelli et al., Nucl. Instrum. Methods Phys. Res., Sect. A 506, 250 (2003).

[40] T. Rzaca-Urban et al., Eur. Phys. J. A 9, 165 (2000).

[41] N. Paul et al., Phys. Rev. Lett. 118, 032501 (2017).

[42] S. Chen et al., Phys. Rev. C 95, 041302(R) (2017).

[43] C. Santamaria et al., Phys. Rev. Lett. 115, 192501 (2015).

[44] J. Dechargé and D. Gogny, Phys. Rev. C 21, 1568 (1980).

[45] J. F. Berger, M. Girod, and D. Gogny, Comput. Phys. Commun. 63, 365 (1991).

[46] M. Girod, J.-P. Delaroche, A. Görgen, and A. Obertelli, Phys. Lett. B 676, 39 (2009).

[47] H. Iwasaki et al., Phys. Rev. Lett. 112, 142502 (2014).

[48] J. Ljungvall et al., Phys. Rev. Lett. 100, 102502 (2008).

[49] J. Dudouet et al., Phys. Rev. Lett. 118, 162501 (2017).

[50] J. M. Yao, K. Hagino, Z. P. Li, J. Meng, and P. Ring, Phys. Rev. C 89, 054306 (2014).

[51] M. Bender, P. Bonche, and P.-H. Heenen, Phys. Rev. C 74, 024312 (2006).

[52] C. Sotty et al., Phys. Rev. Lett. 115, 172501 (2015).

[53] R. Rodriguez-Guzman, P. Sarriguren, and L. M. Robledo, Phys. Rev. C 82, 061302(R) (2010). 